\title{
EXCITON ELECTRO-ABSORPTION AT NON-ZERO WAVE VECTOR
}

\author{
M. Slawik and P. Petelenz \\ K. Gumiński Department of Theoretical Chemistry, Jagiellonian University \\ Ingardena 3, 30-060 Kraków, Poland
}

(Received April 5, 1995; in final form June 12, 1995)

\begin{abstract}
Dedicated to Professors Krzysztof Pigoń, Józef W. Rohleder and Zdzisław Ruziewicz on the occasion of their 70th birthdays*
\end{abstract}

Based on a previously proposed two-dimensional model of a polyacene crystal, the contributions to the electro-absorption signal from different points of the exciton Brillouin zone are calculated. They are shown to differ substantially both in amplitude and in shape. This makes electro-absorption spectra very sensitive to vibronic coupling. On the one hand, the new factor is a potential complication in theoretical interpretations. On the other hand, it favours electro-absorption spectroscopy as a promising experimental tool to study vibronic effects.

PACS numbers: $78.30 . \mathrm{Jw}, 78.20 .-\mathrm{e}, 78.40 .-\mathrm{q}$

\section{Introduction}

In absorption spectroscopy of molecular crystals, the effect of vibronic coupling with high-frequency totally symmetric intramolecular vibrations is twofold. Firstly, an electronic transition is accompanied by a Franck-Condon progression of vibrational satellites, which in crystal spectra can be usually quite well resolved. Secondly, the coupling to phonons results in the relaxation of the $\boldsymbol{k}=\mathbf{0}$ optical selection rule in the exciton subspace. The true eigenstates of the crystal correspond to excitonic polarons where an exciton is bound to a cloud of virtual phonons. As the phonons carry some wave vector (quasi-momentum), the exciton engaged in the optically allowed $k=0$ excitonic polaron may itself have a non-zero wave vector. This allows one to probe optically the states from the entire exciton Brillouin zone, with weights depending on the strength of vibronic coupling and on the density of exciton states throughout the zone. In the spectrum, this results in the broadening of the corresponding line and may give rise to phonon side bands [1].

${ }^{*}$ Submitted on invitation of the Institute of Physical and Theoretical Chemistry, Technical University of Wroclaw, Wroctaw, Poland. 
The above effect occurs for the $v=0$ state $(0-0$ line $)$ as well as for its vibronic replicas. In the latter case, however, real phonons may participate in addition to the virtual ones, so that e.g. the $v=0$ and $v=1$ polarons should normally have different phonon contributions, and may effectively probe different parts of the exciton Brillouin zone [2].

As a general solution of the vibronic problem in a molecular crystal is very difficult, it was done only for one dimension. The most complete treatment is due to Scherer and Fischer [2]. They have shown that the distribution of absorption intensity over the exciton Brillouin zone is quite different for $v=0$ and $v=1$, in the latter case most of the intensity being concentrated at zone ends.

However, the possible variations of the absorption spectrum induced by vibronic coupling are limited by the fact that absorption intensities are positive definite. Although new bands may appear (at non-zero exciton wave vector [2]), but normally most of the effect shows up only as band broadening or minor changes of the shape.

This need not be the case in electro-absorption (EA) spectroscopy, where the signal has a derivative shape and may have either positive or negative values. The spectrum is generally much more sensitive even to minor changes of individual contributions. The Frenkel and charge transfer (CT) states may be differently affected by vibronic coupling, and the contributions from different regions of the Brillouin zone may also substantially differ. An extreme case of this view was presented in Ref. [3], where one of us suggested CT electro-absorption in centrosymmetric crystals to be an inherently vibronic phenomenon, with non-zero contribution only from the states located at the edges of the exciton Brillouin zone.

It was demonstrated [3] that the second derivative contributions to the EA spectrum must be attributed to degenerate states. As such states appear at the edge of the exciton Brillouin zone, that particular region seemed to be favoured by this selection rule.

It was shown later [4] that the relevant states need not be exactly degenerate, and that it is the spectral width of the bands (usually substantial) that sets the energy scale according to which the states should be considered as quasi-degenerate. This allows many states, both Frenkel and CT, from various regions of the Brillouin zone, to be EA-active.

An analysis of the EA contributions from the centre of the exciton Brillouin zone is the obvious starting point, and is presently being completed [5]. However, the role of off-centre contributions, allowed due to vibronic coupling, has also to be assessed.

In order to describe the EA spectra of simple molecular crystals like polyacenes, a two-dimensional model is needed [6]. It is also necessary to include the coupling between the Frenkel and CT excitations [3]. This seems to preclude advanced treatment of vibronic effects, since direct application of the Scherer-Fischer approach (one dimension, one exciton state per molecule) is not possible, while its major generalization is at the moment numerically inaccessible.

On that view, it seems useful to invert the problem. Instead of developing a sophisticated description of vibronic effects, we will attempt to find out how much the EA contributions from different parts of the Brillouin zone really differ. This 
will give us indirect information as to the possible changes in the EA spectrum that would result if the off-centre contributions are activated by vibronic coupling.

\section{Model}

Our treatment is based on the previously proposed [6] two-dimensional model of Frenkel and CT excitations in polyacene crystals. The model includes the nearest-neighbour off-diagonal coupling in the $(0,1,0)$ and $( \pm 1 / 2, \pm 1 / 2,0)$ crystal directions. In the present context, it is modified by explicit inclusion of the interaction $\Delta(F)$ with the external electric field $F$. In the "local" basis of Ref. [6], the new contributions read

$$
\begin{aligned}
& \left\langle A^{*} B|\Delta(F)| A^{*} B\right\rangle=\frac{1}{2} p F^{2}, \\
& \left\langle A^{+} B^{-}|\Delta(F)| A^{+} B^{-}\right\rangle=\frac{1}{2} p F^{2}-\boldsymbol{m} \cdot \boldsymbol{F},
\end{aligned}
$$

where $A, B$ denote two nearest-neighbour molecules, $p$ is the polarizability change between the ground state and the respective "local" excited state (either Frenkel or CT), and $\boldsymbol{m}$ is the dipole moment (non-zero only for CT states). The anisotropy of polarizability change is neglected; its inclusion would barely affect the results, which are to be directionally a veraged anyway (vide infra).

Energetic effects of vibronic coupling with the $0.17 \mathrm{eV}$ progression-forming intramolecular mode are included by the standard renormalization of the transfer integrals [3], reflecting the vibrational band narrowing. It is necessary to take it into account in order to maintain the salient relationships between different contributions to exciton energy. In view of the qualitative nature of the present study, the quantitative difference between the narrowing factors for $v=0$ and $v=1$ is irrelevant for the parameter values of interest. Therefore, as far as energies are concerned, the conclusions will be applicable both to the 0-0 region and to the first vibronic satellite.

As in Ref. [6], the Hamiltonian is block-diagonalized by means of the Fourier transformation, and subsequently diagonalized numerically for each value of the wave vector $k=\left(k_{a}, k_{b}\right)$. The resulting eigenvectors are used to calculate the absorption intensities. The $k=0$ selection rule is ignored, as presumably violated due to the vibronic coupling.

Absorption to each eigenstate is represented by a Gaussian curve. This is done at zero and at non-zero electric field, and the electro-absorption signal is calculated by subtracting the corresponding two spectra. In order to account for the random distribution of crystallite orientations in polycrystalline or quasi-amorphous samples used in actual experiments [7-10], the calculations are repeated for different orientations of the electric field with respect to crystal axes, and the results averaged. As in most experiments unpolarized light is used, the results are also a veraged over two different light polarizations (electric vector parallel and perpendicular to the modulating external electric field $\boldsymbol{F}$ ).

Effectively, the calculations may yield the electro-absorption signal for any $k=\left(k_{a}, k_{b}\right)$ pair from the exciton Brillouin zone. In reality, only a representative set of $k$ values is probed. It should be noted marginally that the value of $k$ is not related to the wave vector of incident radiation (which, as always, is negligible), but 
of the intramolecular phonon which couples the given state to the $k=0$ exciton. In the present treatment, the vibrational degrees of freedom are not explicitly included, so that the effect of phonons should be viewed merely as a source of weights with which the individual states (characterized by $k$ ) from the exciton Brillouin zone would contribute to the observed EA signal.

In view of the model nature of this study, the actual values of input parameters need not represent any real crystal. In order to fix attention, we chose two sets, representing the anthracene and the tetracene crystal. The numerical values of the parameters were the same as used in Ref. [6] for the vibrationally unexcited $(v=0)$ electronic states. The transition dipole moments of the CT states were adopted according to Refs. [3,11], and the spectral widths of individual states were arbitrarily set at $\sigma=0.02 \mathrm{eV}$, which is of the order of the values obtained in actual experiments $[7,8]$.

For polarizabilities, the free-molecule values were used in tentative calculations. Ilowever, the polarizability contributions to the EA signal turned out to be negligible. Even in the states of Frenkel parentage, they amounted to less than $10 \%$ of the signal which was dominated by the dipole moment term of Eq. (2) (due to the mixing with the CT states) ${ }^{\dagger}$. For this reason, the results are consistently presented for $p$ set equal to zero.

Electric field strength was set at a typical value of $50 \mathrm{kV} / \mathrm{cm}[7,8]$.

According to the experimental geometry used by Sebastian et al. [7], for anthracene the samples were assumed to be polycrystalline, with crystallite $a b$ planes parallel to the substrate and the direction of the $b$ axis completely random with respect to the electric field. The field was parallel to the substrate and to crystallite $a b$ planes. The light was assumed to propagate along the direction perpendicular to the crystallite $a b$ planes (electric vector parallel to the planes).

For tetracene, the calculations were done for quasi-amorphous samples (three-dimensional averaging), again in accordance with the experimental geometry [8]. The results are not shown here, since their salient features are the same as for the anthracene case.

It should be emphasized that the present results are not aimed at direct comparison with experiment. In the first place, the experimental spectra [7-10] contain substantial contributions from vibrational satellites $(v \neq 0)$. Secondly, the recent results [5] indicate that for a quantitative interpretation [5] of the experimental spectra $[7,8]$ it is necessary to include other Frenkel states which, although inactive in one-photon absorption spectroscopy, need not be mute in the EA signal. In the present context, both complications would compel us to use a more general model, unnecessarily obfuscating the main point of the paper.

\section{Results}

The figures show the electro-absorption signals calculated for anthracene for different values of $k_{a}$ at $k_{b}=0$ (Fig. 1) and at different values of $k_{b}$ at $k_{a}=0$ (Fig. 2).

\footnotetext{
In perfect agreement with previous results [12] indicating that the excited state polarizabilities are dominated by nonlocal contributions (coupling of Frenkel excitons to charge transfer states).
} 


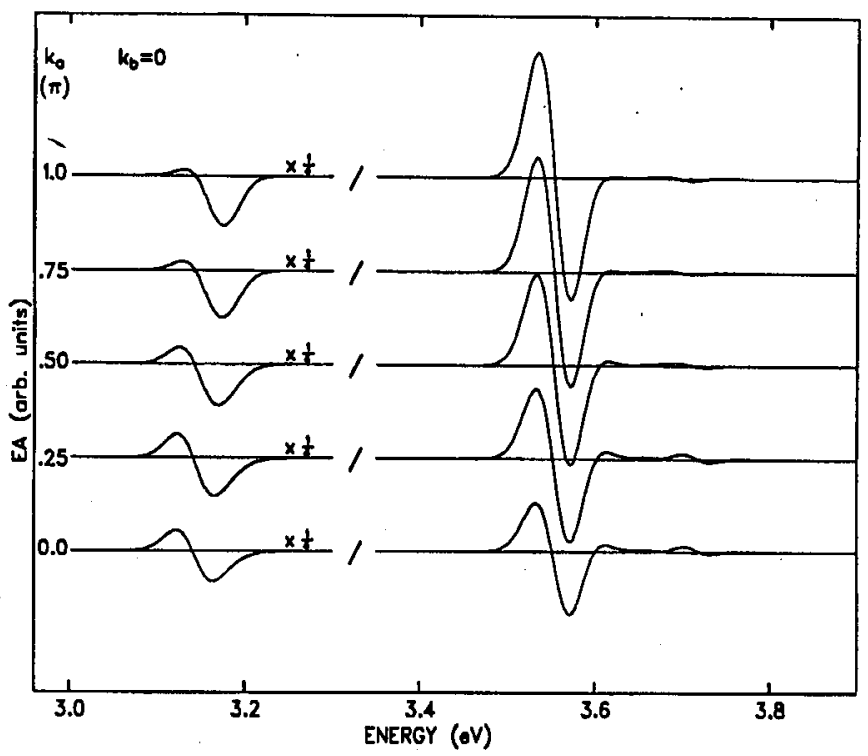

Fig. 1. Calculated EA signal for the anthracene crystal at $k_{b}=0$ and different $k_{a}$ values. In order to facilitate comparison, the intensity of the Frenkel exciton part of the spectrum (around $3.15 \mathrm{eV}$ ) is multiplied by 4; the same rule is applied for Figs. 2 and 3 .

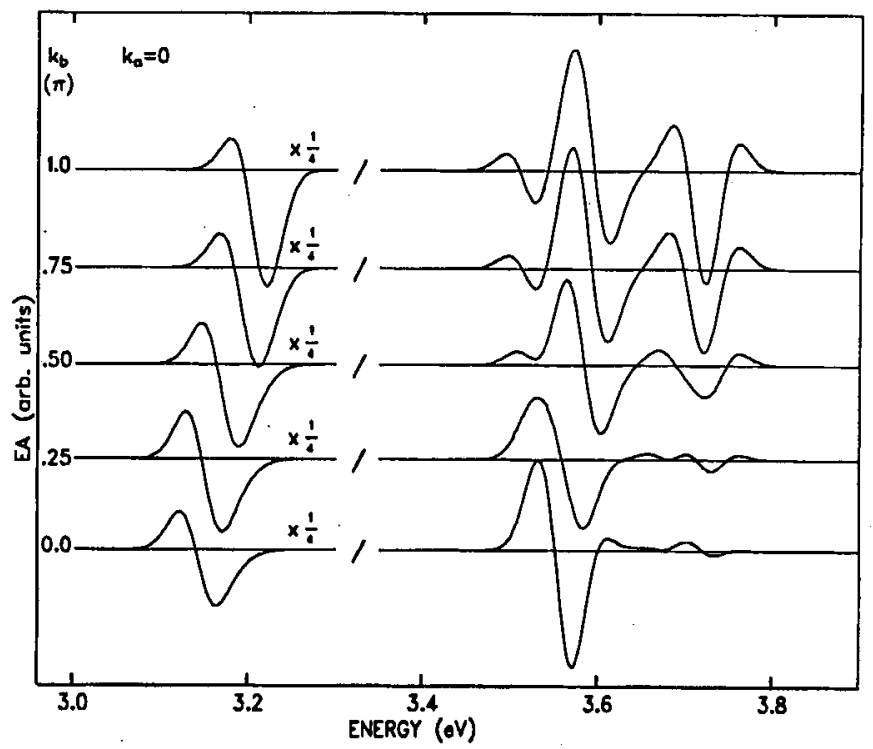

Fig. 2. Calculated EA signal for the anthracene crystal at $k_{a}=0$ and different $k_{b}$ values. 
The first-derivative feature at about $3.15 \mathrm{eV}$ represents the Frenkel state, while the cluster of features at $3.5-3.7 \mathrm{eV}$ corresponds to charge-transfer states (at 3.5-3.6 eV - mostly of $(1 / 2,1 / 2,0)$, and at about $3.7 \mathrm{eV}$ - predominantly of $(0,1,0)$ parentage).

The changes of $k_{a}$ at fixed $k_{b}$ substantially affect the intensities but produce only slight spectral shifts.

The effect of $k_{b}$ changes at fixed $k_{a}$ is much more spectacular. The signal at $3.55 \mathrm{eV}$ has for $k_{b}=0$ a distinct first-derivative shape with a slight second-derivative admixture (small maximum at the high-energy side of the minimum). At larger $k_{b}$ values it transforms into a broader first-derivative feature, acquiring at Brillouin zone edge $\left(k_{b}=1\right)$ a much more complex form whose maximum is located at the position of the minimum observed for $k_{b}=0$. The amplitude of the feature appearing at about $3.7 \mathrm{eV}$, for $k_{b}=0$ barely noticeable, increases by more than an order of magnitude when $k_{b}=1$, while its shape changes from almost pure first derivative to almost pure second derivative.

For tetracene, whose structure is triclinic instead of monoclinic, the changes of the signal along the $k_{a}$ direction are almost as large as along $k_{b}$, which presumably results from the change of lattice symmetry. As there are no other qualitative differences, the tetracene results are not displayed here.

As it has already been mentioned, there is no way to probe experimentally individual points of $k$-vector space: the observable spectrum is a superposition of the contributions from all the Brillouin zone. For the $v=0$ vibrational state, the $k \neq 0$ exciton states gain non-zero intensity from the admixtures of the $k=0$ wave function, mediated by vibronic coupling with the $(v=1, k=0)$ states.

The vibronic coupling with intramolecular phonons is in the direct space strictly localized at the molecule where the exciton resides. Hence, in the reciprocal space the coupling is distributed with equal strengths over all $k$-states from the exciton Brillouin zone. Assuming the phonons to have no dispersion (Einstein model), and neglecting (with respect to the vibrational frequency) the exciton dispersion, perturbation theory makes one expect the contribution from any $\left(k_{a}, k_{b}\right)$ pair to be weighted by the same factor, and the overall weight of all $k \neq 0$ states with respect to the $k=0$ state to be $b^{2} / 2: 1$, where $b$ stands for the dimensionless vibronic distorsion parameter.

The effective displacement parameter $b$ should not differ much for Frenkel and CT states. In fact, although vibronic distortion in an individual molecule is larger for excitation than for ionization (1.57 vs. 1 [13-15]), but in a CT state two molecules are distorted at the same time, instead of one. Therefore we have adopted for all states the uniform value of $b=1.57$, obtained from the absorption spectrum of the isolated molecule $[14,15]$. Figure 3 shows the simulated EA spectrum, obtained in this way, along with its $k=0$ and $k \neq 0$ components.

With respect to the $k=0$ component (which would be the only contribution observed for zero vibronic coupling), the net signal is broader and some of its features are smeared out. The minor maxima at 3.6 and $3.7 \mathrm{eV}$ disappear, and so does the minimum at $3.65 \mathrm{eV}$. This effect is due to the partial cancellation of the positive and negative contributions from the $k=0$ and $k \neq 0$ signals, and would not be possible in absorption spectroscopy, where the signal is positive definite. 


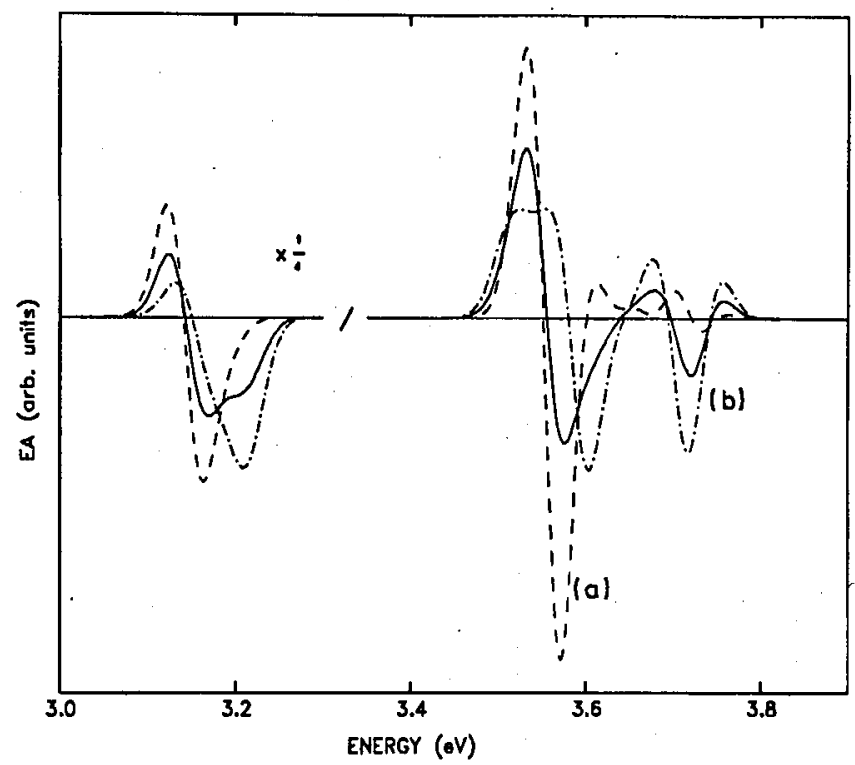

Fig. 3. Simulated EA signal for the anthracene crystal at: (a) $k=0$ (dashed line), (b) averaged over all $k \neq 0$ states with equal weights (dash-dot line); solid line superposition of (a) and (b) with the weights $1: b^{2} / 2$.

In addition, the signal from the CT states of $(0,1,0)$ origin (at about $3.7 \mathrm{eV}$ ) is much more exposed, and its shape is changed from first- to second-derivative. Evidently, a state that is not very prominent at $k=0$ may acquire substantial importance due to vibronic effects.

Worth noting is also the change in the Frenkel exciton region, where the negative lobe is shallower but considerably broader. This is reminiscent of the shapes observed experimentally $[7,8]$ and may suggest vibronic effects as one of the causes of the asymmetry between the positive and negative lobes in Frenkel exciton signals $\ddagger$.

As demonstrated for the simple one-exciton model [2], vibronic effects give rise to a considerable difference in the absorption intensity distribution between the $v=0$ and $v=1$ states: in the former case, the leading contribution comes from the centre of the exciton Brillouin zone $(k=0)$, while in the latter case the contributions from zone edges $(k= \pm \pi)$ predominate. In our present description, this would be represented by different weighting (for $v=0$ and $v=1$ ) of the signals from the zone centre and zone edges.

An extreme effect of such different weighting may be observed by comparing the $\boldsymbol{k}=\mathbf{0}$ and $\boldsymbol{k} \neq \mathbf{0}$ (where the zone edges prominently contribute) EA signals. The dashed line $(k=0)$ should represent the leading contribution to the signal shape for $v=0$; it has a large first-derivative feature with a deep minimum at

$\$$ Vibronic coupling with low frequency vibrations (unresolved in the spectrum) may be another reason. It will be treated in a forthcoming paper. 
about $3.55 \mathrm{eV}$. On the other hand, the dash-dot curve, which (when shifted by one vibrational quantum to higher energies) should roughly mimic the $v=1$ signal; exhibits two rather widely spaced minima (at $3.6 \mathrm{eV}$ and about $3.7 \mathrm{eV}$ ) of almost equal depth. The position of neither of the minima coincides with that predicted for $v=0$ ( $k=0$ curve), and the overall shape suggests large second-derivative contributions.

Direct quantitative relevance of the above observations is open to discussion, since they are based on the extrapolation of the conclusions of Ref. [2] from a one- to a two-dimensional model, and from one to a number of exciton states. Nevertheless, the results do suggest that the shape of the electro-absorption signal of a vibronic satellite may substantially differ from its $v=0$ counterpart, while in previous analyses $[7,8,3]$ similar shapes were assumed by default.

\section{Discussion}

There are many level crossing points in the Brillouin zone, and at different $k$ values the eigenstate of the same parentage may have different Frenkel and CT contributions. Therefore, in the present context, (1) the degeneracies (crucial in EA spectroscopy $[3,4])$ had to be handled with special care, and (2) the "primarily Frenkel" or "primarily CT" character could not be arbitrarily attributed to the eigenstates.

For these reasons, the calculations are based on a new methodology.

For the first time, the two-dimensional model of Ref. [6] is harnessed to generate the electro-absorption spectrum. In contrast to all models used previously for this purpose [3,6-11], it accounts correctly for the symmetry (and hence degeneracy) of the CT states.

Secondly, the simulated EA spectrum is generated in an entirely new way. In previous studies on polyacene electro-absorption [3,6-11] the spectrum was synthesized from the first-derivative contribution (mostly taken from experiment) representing mostly the Frenkel states, and from a set of second-derivative curves ascribed to individual CT states. The results of Ref. [4] indicate that the actual CT contributions need not have second-derivative shape. On the other hand, the eigenstates of Frenkel parentage, having now some CT character, may acquire considerable second-dervative admixtures. Hence, there is little substance in the traditional way of attributing distinct spaectral features to particular types of states. The present approach avoids this difficulty: the spectrum is generated directly by subtracting the simulated absorption spectra at zero and non-zero electric field; the first- or second-derivative shape emerges as a conclusion rather than a postulate.

Having eliminated the potential source of artefacts (as described above), our treatment produced the following conclusions.

At different points of the Brillouin zone the exciton states have different energies, but also the shapes of their EA signals may substantially differ. As their contributions to the observed electro-absorption spectrum are weighted by vibronic effects, the above results highlight the importance of these effects in the context of EA spectroscopy. 
Accordingly, there is little hope that the description of EA spectra that has focused so far on the purely electronic aspects of the problem [7, 8, 3-5], may produce quantitatively correct results, especially in the energy region where vibronic replicas dominate. Either we have to reduce our expectations as to the agreement of the reproduced spectra with experiment, or a reasonably complete vibronic description has to be developed. As this is a very difficult task, the present conclusion is in a way pessimistic.

However, it has also a positive aspect. Sensitivity of the EA signal to vibronic eflects turns electro-absorption spectroscopy into a potentially promising tool for studying vibronic coupling. When combined with numerical band-shape analysis techniques, it should be superior to absorption spectroscopy in probing (indirectly but effectively) vibronic effects at exciton wave vector different from zero, and in this way provide a stringent test for theoretical models. This potential has yet to be explored.

\section{References}

[1] V.M. Agranovich, Theory of Excitons, Nauka, Moscow 1968.

[2] P.O.J. Scherer, S.F. Fischer, Chem. Phys. 86, 269 (1984).

[3] P. Petelenz, V.H. Smith, Jr., Chem. Phys. 131, 409 (1989).

[4] P. Petelenz, Chem. Phys. 171, 397 (1993).

[5] P. Petelenz, M. Slawik, in preparation.

[6] P. Petelenz, M. Slawik, Chem. Phys. 157, 169 (1991).

[7] L. Sebastian, G. Weiser, G. Peter, H. Bässler, Chem. Phys. 75, 103 (1983).

[8] L. Sebastian, G. Weiser, H. Bässler, Chem. Phys. 61, 125 (1981).

[9] J. Kalinowski, W. Stampor, B. Petelenz, P. Petelenz, Chem. Phys. 167, 185 (1992).

[10] W. Stampor, Ph.D. Thesis, Technical University of Gdańsk, Gdańsk 1994.

[11] R.W. Munn, P. Petelenz, W. Siebrand, Chem. Phys. 111, 209 (1987).

[12] P. Petelenz, Chem. Phys. Lett. 215, 607 (1993).

[13] P.A. Clark, F. Brogli, E. Heilbronner, Helv. Chim. Acta 55, 1415 (1972).

[14] D.W. Schlosser, M.R. Philpott, Chem. Phys. 49, 187 (1980).

[15] M.R. Philpott, J. Chem. Phys. 50, 5117 (1969). 